\title{
CARACTERÍSTICAS FíSICAS E QUíMICAS DE SUBSTRATOS À BASE DE PÓ DE COCO E RESíduO de SiSAl PARA PRODUÇÃo de MUdAS de SABIÁ (Mimosa caesalpiniaefolia Benth) $^{1}$
}

\author{
Maria Regina Beltrão Lacerda ${ }^{2}$, Marco A. A. Passos ${ }^{3}$, José Júlio V. Rodrigues ${ }^{4}$ e Levy P. Barreto
}

\begin{abstract}
RESUMO - O experimento foi instalado com o objetivo de analisar as características físicas e químicas ocorridas em substratos com diferentes concentrações de pó de coco (PC), resíduo de sisal (RS) e Argissolo VermelhoAmarelo distrófico (Arg), no crescimento de mudas de sabiá (Mimosa caesalpiniaefolia Benth), produzidas em recipientes com capacidade para $2 \mathrm{dm}^{3}$. O delineamento utilizado foi o inteiramente casualizado, com 13 tratamentos e quatro repetições, contendo oito vasos por repetição, num total de 416 unidades amostrais. Avaliaram-se as características físicas e químicas, bem como as variáveis da altura total das plantas e do diâmetro do coleto a cada 30 dias, a área foliar, o número de folhas e a massa seca da parte aérea. Recomenda-se o uso de pó de coco como componente para substratos, pois suas propriedades físicas e químicas, aliadas à sua estrutura e durabilidade, apresentaram condições para a produção de mudas de sabiá. Não se recomenda o uso do resíduo de sisal como componente para substratos na produção de mudas de sabiá, pois esse resíduo revelou valores de condutividade elétrica (CE) e pH inadequados ao cultivo.
\end{abstract}

Palavras-chave: Características físicas e químicas, sabiá, Mimosa, substratos, pó de coco e resíduo de sisal.

\section{PHYSICAL AND CHEMICAL CHARACTERISTICS OF COIR DUST AND SISAL RESIDUE FOR SEEDLING PRODUCTION OF SABIÁ (Mimosa casalpiniaefolia Benth)}

\begin{abstract}
An experiment was carried out to analyze the physical and chemical characteristics of substrates with different concentrations of coir dust (PC), sisal residue (RS) and dystrophic red-yellow Argissoil (Arg) on the seedling development of 'sabiá' (Mimosa caesalpiniaefolia Benth) in $2 \mathrm{dm}^{3}$ containers. A completely randomized experimental design was used, with 13 treatments, 4 replications and 8 pots per replication, totaling 416 sample units. Physical and chemical characteristics were evaluated, as well as total height and diameter every 30 days, leaf area, number of leaves and dry matter weight. Coir dust was recommended as substrate component for 'sabiá' seedling production due to its physical and chemical properties along with durability and structure. Sisal residue was not recommended as substrate component since it showed unsuitable conditions of electrical conductivity (EC) and pH for seedling production
\end{abstract}

Keywords: Physical and chemical characteristics, 'sabiá', Mimosa, substrates, coir dust, and sisal residue.

\footnotetext{
${ }^{1}$ Recebido em 05.03.2005 e aceito para publicação em 10.11.2005.

${ }^{2}$ Eng $^{a}$. Agrônoma, M.S. Ciência Florestal pela UFRPE, 52171-030 Recife-PE.

${ }^{3}$ Departamento de Ciência Florestal da UFRPE.

${ }^{4}$ Departamento de Agronomia da UFRPE.

${ }^{5}$ Departamento de Química da UFRPE.
} 


\section{INTRODUÇÃO}

A Mimosa caesalpinieaefolia Benth, conhecida como sabiá, sansão-do-campo, é uma espécie nativa da caatinga do Nordeste do Brasil. É uma planta que, além de fornecer madeira para usos como estacas, moirões, lenha e carvão, apresenta características ornamentais e, por ser uma planta pioneira e de rápido crescimento, é muito usada em plantios heterogêneos na recuperação de áreas degradadas (LORENZI, 2002).

Inúmeros substratos em sua constituição original ou combinada são usados atualmente para propagação de espécies florestais via sementes ou vegetativamente. $\mathrm{Na}$ escolha de um substrato, devem-se observar, principalmente, suas características físicas e químicas, a espécie a ser plantada, além dos aspectos econômicos, quais sejam: baixo custo e grande disponibilidade (FONSECA, 2001).

Dificilmente se encontra um material com todas as características para atender às condições para o ótimo crescimento e desenvolvimento das plantas (SOUZA et al., 1995). Nesse sentido, o substrato poderá ser formado de solo mineral ou pode ser orgânico, de um ou de diversos materiais ou misturas, como a casca de arroz (in natura, carbonizada ou queimada), poliextrileno expandido (isopor), espuma fenólica, areia, produtos da madeira, como serragem e maravalha, compostos de lixo domiciliar urbano, compostos de restos de poda, vermicomposto, fibra de coco semidecomposta e lã-de-rocha (VERDONCK, 1984; FONTENO, 1996; BURGER et al., 1997; PUCHALSKI, 1999; SCHIE, 1999; KÄMPF, 2000).

Negreiros et al. (2004) salientaram a conveniência da associação de materiais orgânicos, especialmente em mistura com o solo, para melhorar a textura do substrato e, dessa maneira, propiciar boas condições físicas e fornecer os nutrientes necessários ao desenvolvimento das raízes e da muda.

Entre as propriedades físicas mais importantes, encontram-se a densidade do substrato, a porosidade total, o espaço de aeração e a retenção de água. Segundo Kämpf (2000), quanto mais alta a densidade, mais difícil fica o cultivo no recipiente, quer por limitações no crescimento ou pelo custo do transporte dos vasos ou bandejas.

Solos e substratos são meios porosos, formados por sólidos e poros preenchidos por água e ar (KÄMPF, 2001). Os poros são responsáveis pelas trocas gasosas

R. Árvore, Viçosa-MG, v.30, n.2, p.163-170, 2006 entre o substrato e a atmosfera, bem como determinam os movimentos da água no vaso e a drenagem. Portanto, entender a dinâmica das relações entre sólidos e os poros é fundamental para se obter sucesso na produção de mudas.

As características químicas mais importantes nos substratos são: $\mathrm{pH}$ e a condutividade elétrica (SILVEIRA et al., 2002). Com relação ao pH, os substratos devem apresentar valores dentro de uma faixa considerada adequada para o cultivo de plantas, pois valores inadequados, além de influenciar a disponibilidade de nutrientes (CARNEIRO, 1995), estão relacionados a desequilíbrios fisiológicos (WILSON, 1983). Conforme Kämpf (2000), em substratos onde predomina a matéria orgânica a faixa ideal de $\mathrm{pH}$ recomendada é de 5,0 a 5,8 e, quando for à base de solo mineral, entre 6,0 e 6,5 .

Outra propriedade relevante na composição de meios de cultivo é a salinidade que pode ser derivada da adubação de base, do conteúdo natural de sais presentes nos componentes utilizados na mistura e, ainda, pelo uso de misturas excessivamente ricas em nutrientes, uma vez que o excesso de sais pode prejudicar o crescimento das plantas (GRAZIANO et al., 1995; HANDRECK e BLACK, 1999).

No mundo todo, a indústria de substratos para plantas busca materiais mais bem elaborados e que atendam às necessidades das plantas e dos produtores. Resíduos da agroindústria, como o pó de coco e os materiais orgânicos decompostos, aparecem como alternativas promissoras para as misturas (K ̈̈̈MPF, 2000).

Pó de coco é o nome dado ao resíduo oriundo do material fibroso que constitui o mesocarpo do fruto do coqueiro (Cocus nucifera L.), de onde são retiradas fibras longas utilizadas na fabricação de cordas, tapetes e muitos outros produtos; desse processamento resultam uma mistura de fibras curtas e uma considerável quantidade de pó. O pó de coco possui grande porcentagem de lignina (35-45\%) e de celulose (23$43 \%$ ) e uma pequena quantidade de hemicelulose (3$12 \%$ ), que é a fração vulnerável ao ataque de microrganismos. Essas características conferem ao substrato de fibra de coco grande durabilidade, sendo, dessa maneira, recomendável para cultivos de ciclo longo como ornamentais, assim como o cultivo de hortícolas sem o solo, pois não sofre o processo de 
degradação acelerado causado pela intensa aplicação de água e fertilizantes (NOGUERA et al., 2000). Conforme Teo e Tan (1993), a estrutura do pó de coco associado às suas propriedades físico-químicas torna-o particularmente adequado para ser utilizado como substrato.

O sisal (Agave sisalana Perrine) é uma planta eminentemente tropical, e por isso existem tão poucos plantios comerciais com essa fibrosa fora desse ambiente. Pertence à família Agavaceae, genêro Agave e espécie sisalana. A planta é originária do México que produz a mais importante fibra dura do mundo, sendo bem adaptada ao clima predominante no Nordeste do país. No Brasil, a produção concentrase principalmente nos Estados da Bahia e da Paraíba, hoje os dois maiores produtores. O resíduo de sisal é oriundo do desfibramento da parte fibrosa da folha, constituído de pedaços de folhas e fibras de diferentes tamanhos (MOREIRA et al., 1996).

A necessidade de caracterizar materiais encontrados nas diferentes regiões do país e torná-los disponíveis como substrato agrícola é fundamental, pois, além de ser uma alternativa para reduzir os custos de produção, daria destino ao resíduo acumulado (ANDRIOLO, 1999). Assim, o trabalho teve como objetivos caracterizar as propriedades físicas e químicas dos componentes dos substratos formulados à base de pó de coco e resíduo de sisal em diferentes combinações no crescimento inicial das mudas de sabiá (Mimosa caesalpiniaefolia Benth) cultivadas em viveiro.

\section{MATERIAL E MÉTODOS}

O experimento foi instalado e conduzido no viveiro do Departamento de Ciência Florestal da Universidade Federal de Pernambuco (UFRPE). Foram formulados 13 substratos compostos por pó de coco (PC), resíduo de sisal (RS) e Argissolo Vermelho-Amarelo distrófico (Arg) com as seguintes combinações volumétricas: (PC) $100 \%,(\mathrm{RS}) 100 \%,(\mathrm{Arg}) 100 \%, \operatorname{Arg}+\mathrm{PC}(1: 1 ; 1: 2$; $1: 4), \operatorname{Arg}+\mathrm{RS}(1: 1 ; 1: 2 ; 1: 4)$ e Arg+PC+RS (1:1:1;1:2:2; $1: 2: 4 ; 1: 4: 2)$. O delineamento experimental adotado foi o inteiramente casualizado, com 13 tratamentos e quatro repetições, contendo oito vasos por repetição, perfazendo um total de 416 unidades amostrais.

O pó de coco foi proveniente de uma fábrica de beneficiamento de coco maduro, e o resíduo de sisal foi proveniente do CNPA-EMBRAPA, de Campina Grande, PB.
As sementes de sabiá (Mimosa caesalpiniaefolia Benth) foram beneficiadas e submetidas a um tratamento pré-germinativo com ácido sulfúrico concentrado durante $1 \mathrm{mim}$, para promover maior uniformidade de germinação. Logo após foram lavadas em água corrente e, em seguida semeadas (13/10/2003), sendo quatro sementes por vasos, com capacidade para $2 \mathrm{dm}^{3}$. Após 15 dias do plantio, realizou-se o desbaste, deixando uma planta por vaso.

Nos substratos formulados não se realizou correção. A adubação foi em cobertura efetuada com solução aquosa, a base de $\mathrm{N}$ e de $\mathrm{K}$ a $100 \mathrm{mg} / \mathrm{dm}^{3}$ e de 200 $\mathrm{mg} / \mathrm{dm}^{3}$ de $\mathrm{P}$ em dose única e da solução de Norris (1976) contendo micronutrientes para leguminosas, parcelados em intervalos de 15 dias. As mudas foram regadas diariamente.

A densidade das partículas (Dp) seguiu a metodologia da Embrapa (1997), com modificação. Utilizou-se um balão volumétrico de $100 \mathrm{~mL}$, e adicionou-se álcool etílico. Os cálculos foram obtidos através da fórmula:

$$
D p=\frac{a}{100-b}
$$

em que $a$ é o peso da amostra seca a $105^{\circ} \mathrm{C}$ e $b$, o volume de álcool gasto.

A Capacidade de aeração (CA), Capacidade de recipiente $(\mathrm{CR})$ na base de massa e de volume, Densidade global (Dg), Porosidade total efetiva (PTe) e Porosidade total teórica $(\mathrm{PTt})$ foram determinadas, utilizando-se copos plásticos com capacidade para $500 \mathrm{~cm}^{3}$ e com $12 \mathrm{~cm}$ de altura contendo orifícios na base, com três repetições para cada substrato, conforme metodologia de Pragana (1999).

Com a saturação completa dos substratos, todos os poros foram preenchidos com água. O volume de água que drena livremente corresponde aos macroporos. Esse volume dividido pelo volume total do recipiente corresponde à Capaciade de recipiente $(\mathrm{CA})$, dada em porcentagem. A Densidade (Dg) foi obtida pela divisão do peso do substrato secado em estufa (PSS) a 105 ${ }^{\circ} \mathrm{C}$, por um período de $24 \mathrm{~h}$, pelo volume total do recipiente.

A capacidade de recipiente na base de massa foi obtida pela fórmula:

$$
\theta m(C R)=\left[\left(\frac{P S U-P S S}{P S S}\right) 100\right]
$$

R. Árvore, Viçosa-MG, v.30, n.2, p.163-170, 2006 
sendo $\theta \mathrm{m}$ a umidade na base de massa; $P S U$ o peso do substrato úmido depois de cessada a drenagem e $P S S$, o peso do substrato secado em estufa.

A capacidade de recipiente na base de volume foi obtida pela fórmula:

$$
\theta v=(C R)=\theta m(C A) \cdot D g
$$

em que: $\theta v=$ umidade na base de volume;

$\theta \mathrm{m}=$ umidade na base de massa $; \mathrm{e}$

$\mathrm{Dg}=$ densidade global.

A Porosidade total efetiva (PTe) foi determinada pela soma do volume de água que ocupava os macroporos e drenado livremente após a saturação (capacidade de aeração), mais o volume de água retido na capacidade de recipiente dividido pelo volume total da amostra. A porosidade total foi obtida pela fórmula:

$$
P T e=1-\frac{D g}{D p}
$$

Para determinação da Condutividade Elétrica (CE) e do $\mathrm{pH}$ dos substratos foi adotada a metodologia citada por Ingram et al. (1990), através do procedimento da diluição volumétrica $2: 1$. O pH foi mensurado em potenciômetro e a condutividade elétrica, com condutivímetro, sendo a CE expressa em dS/m.

Nas avaliações biológicas, obtiveram-se a altura das plantas (h), o diâmetro do coleto (D) em intervalos de 30 dias e o peso da matéria seca da parte aérea (MSPA); a contagem do número de folhas (NF) aos 90 dias após o plantio, segundo Beninncasa (1988).

A área foliar (AF) foi determinada segundo o método descrito por Paulilo e Felippe (1992), modificado. Foram retiradas seis folhas de cada planta, sendo duas da base, duas do meio e duas apicais, todas já desenvolvidas. Foram desenhados os contornos delas em papel de densidade homogênea (papel-ofício tipo A4) e recortados os papéis com os contornos das folhas. Comparouse o peso das folhas cortadas, com o peso médio de 10 quadrados de $2 \mathrm{~cm}^{2}(2 \times 1)$ desenhados no mesmo papel.

Os resultados obtidos das características físicas, químicas e de crescimento das plantas foram submetidos à análise da variância, e as diferenças entre as médias foram comparadas pelo teste de Tukey a $5 \%$ de probabilidade.

R. Árvore, Viçosa-MG, v.30, n.2, p.163-170, 2006

\section{RESULTADOS E DISCUSSÃO}

Os substratos PC e RS apresentaram menores médias de densidade global (Dg) e densidades das partículas (Dp), provavelmente por serem orgânicos e também não diferiram estatisticamente entre si. O substrato Arg apresentou médias superiores de Dg e Dp, em comparação com os demais substratos, aliados à baixa capacidade de retenção de umidade, de aeração e porosidade, exercendo, assim, influência nas demais combinações em que esteve presente (Tabela 1).

Segundo De Boodt e Verdonck (1972), quanto maior a densidade aparente, maior a compactação, menor estrutura e menor porosidade total, sendo maiores as restrições para o crescimento e desenvolvimento das plantas.

Tillmann et al. (1994) encontraram Dg de 0,12 $\mathrm{kg} / \mathrm{dm}^{3}$ para a vermiculita e Rodrigues et al. (1995), valores superiores ao da pesquisa sobre um composto de pó de coco e vermiculita, cuja $\mathrm{Dg}$ de 0,32 e 0,29 $\mathrm{kg} / \mathrm{dm}^{3}$ e de Dp de $2,14 \mathrm{~kg} / \mathrm{dm}^{3}$ e $2,86 \mathrm{~kg} / \mathrm{dm}^{3}$, respectivamente; entretanto, Santos (2002) encontrou um valor inferior ao da pesquisa, cuja Dp é de 1,36 $\mathrm{kg} / \mathrm{dm}^{3}$ para o substrato pó de coco.

O substrato que apresentou o maior valor de $\theta \mathrm{v}$ (CR) foi o PC $(61,85 \%)$, porém não diferiu significativamente, a $5 \%$ de probabilidade, dos tratamentos RS e Arg+PC (1:4), com $\theta \mathrm{v}(\mathrm{CR})$, que foi de $(56,10 \%)$ e de $(50,58 \%)$, respectivamente. O menor valor foi observado no substrato Arg, que foi de $25,52 \%$, não havendo diferença significativa nos tratamentos Arg+RS (1:1) e Arg+RS (1:4).

Valores semelhantes aos encontrados no presente trabalho foram observados por Rodrigues et al. (1995), que encontraram uma $\theta \mathrm{v}(\mathrm{CR})$ de 64,8 e $70,3 \%$ nos substratos pó de coco e vermiculita, respectivamente.

Os substratos RS e Arg+RS (1:4) apresentaram valores de CA muito superiores, sendo 27,71 e 26,25\%, respectivamente. A adição do resíduo de sisal ao solo, na proporção de 1:2 e 1:4, assim como sua adição ao solo e ao pó de coco nas proporções de $1: 2: 2 ; 1: 2: 4$; e 1:4:2, elevou a (CA) dos substratos. O substrato Arg apresentou média de $3,19 \%$, seguido dos substratos Arg+PC (1:1 e 1:2), com exceção dos substratos RS, $\operatorname{Arg}+\mathrm{RS}(1: 2 ; 1: 4), \mathrm{e} \operatorname{Arg}+\mathrm{PC}+\mathrm{RS}(1: 2: 2 ; 1: 2: 4)$, conforme Tabela 1. Resultados divergentes de CA foram obtidos por Pragana em (1999), que encontrou no substrato pó de coco uma CA de $3,3 \%$, portanto um valor abaixo do encontrado neste estudo, que foi de $11,76 \%$ para o substrato PC (Tabela 1). 
Tabela 1 - Densidade do solo (Dg), densidade das partículas (Dp), capacidade de recipiente na base de volume $\theta \mathrm{v}(\mathrm{CR})$, capacidade de aeração (CA), porosidade total efetiva (PTe) e porosidade total teórica (PTt) dos substratos para produção de mudas de sabiá. Recife,PE, 2004

Table 1 - Bulk density (Dg), Particle density (Dp), Volume pot capacity $\theta v(C R)$, Air capacity (AC), Total effective pore space (PTe), Total theoretical pore space (PTt) of substrates for 'sabiá'seedling production. Recife-PE, 2004

\begin{tabular}{lllllll}
\hline Substratos & $\begin{array}{c}\mathrm{Dg} \\
\mathrm{kg} / \mathrm{dm}^{3}\end{array}$ & $\begin{array}{c}\mathrm{Dp} \\
\mathrm{kg} / \mathrm{dm}^{3}\end{array}$ & $\begin{array}{c}\theta \mathrm{v}(\mathrm{CR}) \\
\%\end{array}$ & $\begin{array}{c}\mathrm{CA} \\
\%\end{array}$ & $\begin{array}{c}\mathrm{PTe} \\
\%\end{array}$ & $\begin{array}{c}\mathrm{PTt} \\
\%\end{array}$ \\
\hline Arg* & $1,47 \mathrm{a}$ & $2,65 \mathrm{a}$ & $25,52 \mathrm{~g}$ & $3,19 \mathrm{~d}$ & $28,71 \mathrm{~g}$ & $44,24 \mathrm{~g}$ \\
PC* $^{*}$ & $0,07 \mathrm{f}$ & $1,47 \mathrm{c}$ & $61,85 \mathrm{a}$ & $11,76 \mathrm{bcd}$ & $73,61 \mathrm{ab}$ & $94,33 \mathrm{a}$ \\
RS* & $0,12 \mathrm{f}$ & $1,65 \mathrm{c}$ & $56,10 \mathrm{ab}$ & $27,71 \mathrm{a}$ & $83,81 \mathrm{a}$ & $92,52 \mathrm{a}$ \\
Arg+PC $(1: 1)$ & $0,92 \mathrm{~b}$ & $2,60 \mathrm{a}$ & $37,35 \mathrm{def}$ & $4,77 \mathrm{~d}$ & $42,12 \mathrm{f}$ & $64,25 \mathrm{ef}$ \\
Arg+PC $(1: 2)$ & $0,70 \mathrm{~cd}$ & $2,43 \mathrm{a}$ & $48,72 \mathrm{bcd}$ & $4,78 \mathrm{~d}$ & $53,5 \mathrm{def}$ & $70,93 \mathrm{cdef}$ \\
Arg+PC $(1: 4)$ & $0,45 \mathrm{e}$ & $2,44 \mathrm{a}$ & $50,58 \mathrm{abc}$ & $9,28 \mathrm{~cd}$ & $59,86 \mathrm{cde}$ & $81,04 \mathrm{bc}$ \\
Arg+RS $(1: 1)$ & $0,93 \mathrm{~b}$ & $2,25 \mathrm{a}$ & $33,47 \mathrm{efg}$ & $17,07 \mathrm{abcd}$ & $50,54 \mathrm{ef}$ & $60,94 \mathrm{f}$ \\
Arg+RS $(1: 2)$ & $0,80 \mathrm{bc}$ & $2,32 \mathrm{ab}$ & $37,27 \mathrm{def}$ & $20,98 \mathrm{abc}$ & $58,25 \mathrm{cde}$ & $65,47 \mathrm{def}$ \\
Arg+RS $(1: 4)$ & $0,55 \mathrm{de}$ & $2,30 \mathrm{ab}$ & $29,75 \mathrm{fg}$ & $26,25 \mathrm{a}$ & $56,00 \mathrm{de}$ & $75,41 \mathrm{cde}$ \\
Arg+PC+RS $(1: 1: 1)$ & $0,72 \mathrm{~cd}$ & $2,43 \mathrm{a}$ & $41,61 \mathrm{cde}$ & $9,89 \mathrm{~cd}$ & $51,50 \mathrm{ef}$ & $70,33 \mathrm{cdef}$ \\
Arg+PC+RS $(1: 2: 2)$ & $0,52 \mathrm{de}$ & $2,27 \mathrm{ab}$ & $45,80 \mathrm{bcd}$ & $19,19 \mathrm{abc}$ & $64,99 \mathrm{bcd}$ & $76,98 \mathrm{~cd}$ \\
Arg+PC+RS $(1: 2: 4)$ & $0,42 \mathrm{e}$ & $2,35 \mathrm{ab}$ & $44,49 \mathrm{cde}$ & $24,78 \mathrm{ab}$ & $69,27 \mathrm{bc}$ & $82,05 \mathrm{bc}$ \\
Arg+PC+RS $(1: 4: 2)$ & $0,46 \mathrm{e}$ & $2,14 \mathrm{abc}$ & $46,87 \mathrm{bcd}$ & $15,59 \mathrm{abcd}$ & $62,46 \mathrm{bcde}$ & $78,29 \mathrm{c}$ \\
\hline CV $(\%)$ & 10,73 & 10,96 & 9,06 & 31,17 & 7,56 & 5,43 \\
\hline
\end{tabular}

Médias seguidas da mesma letra na coluna não diferem estatisticamente entre si, a 5\% de probabilidade, pelo teste de Tukey.* Arg-solo, PC-pó de coco e RS-resíduo de sisal.

De acordo com Miner (1994), a capacidade de recipiente e de aeração está correlacionada com a porosidade, e deve haver distribuição adequada entre macro e microporos, permitindo, dessa maneira, que o substrato retenha quantidades convenientes de água e ar.

Os substratos orgânicos pó de coco e o resíduo de sisal apresentaram as maiores porosidades e não diferiram estatisticamente entre si. O tratamento Arg exibiu a menor PTe entre os substratos, como também menores valores para CA de $3,19 \%$ e de $\theta \mathrm{v}(\mathrm{CR})$ de $25,52 \%$, o que justifica uma PTe mais baixa. De maneira geral, as porosidades das combinações de substratos apresentaram valores mais baixos que a dos componentes isolados, exceto o solo, conforme a Tabela 1, provavelmente devido à intermistura de partículas com diâmetros diferentes.

Pragana (1999) obteve resultados semelhantes, encontrando uma porosidade efetiva de $86,05 \%$ para um substrato à base de composto orgânico e uma porosidade total teórica de $93,54 \%$. Resultados análogos foram obtidos por Grás (2000) com substrato à base de perlita, cuja porosidade efetiva foi de $81,3 \%$ e a porosidade total teórica, de $94,9 \%$.

Os substratos PC e RS apresentaram maior PTt, 94,33 e $92,52 \%$, respectivamente, e conseqüentemente menores valores de Dp e Dg. Observa-se na Tabela 1 que o substrato Arg apresentou maiores valores para a Dp de $2,65 \%$ e para a Dg $1,47 \%$, sendo a PTt de $44,24 \%$ a mais baixa entre todas as combinações, uma vez que a porosidade está relacionada com a Dg e a Dp dos substratos. A PTt é maior que a PTe, dada à influência dos poros oclusos na determinação desta última.

Os substratos que promoveram maior crescimento das mudas foram Arg+PC $(1: 1 ; 1: 2 ; 1: 4)$, provavelmente por terem apresentado $\mathrm{CE}$ e $\mathrm{pH}$ mais baixos, conforme a Tabela 2, aliados também a um menor valor de densidade do solo (Tabela 1), e os substratos Arg e PC exibiram médias semelhantes às das combinações anteriormente citadas, conforme se pode observar na Tabela 3.

Com relação ao $\mathrm{pH}$, os substratos $\mathrm{RS}, \mathrm{Arg}+\mathrm{RS}$ $(1: 1 ; 1: 2 ; 1: 4)$ e Arg+PC+RS (1:1:1; $1: 2: 2 ; 1: 2: 4 ;$ e $1: 4: 2)$ apresentaram médias semelhantes, não diferindo estatísticamente (Tabela 2). Resultados semelhantes foram obtidos por Santos (2002), trabalhando com o sabiá (Mimosa caesalpiniaefolia Benth) e utilizando pó de coco como substrato, ou seja, valores de 6,2 para o $\mathrm{pH}$ e 0,42 para CE dS/m. Também, Bezerra et al. (2001), trabalhando com pó de casca de coco maduro, obtiveram valores de $\mathrm{pH}$ de 6,65 e CE de 0,46 dS/m. Os substratos devem apresentar valores adequados de $\mathrm{pH}$ e Condutividade elétrica (CE), uma vez que o

R. Árvore, Viçosa-MG, v.30, n.2, p.163-170, 2006 
$\mathrm{pH}$, além de influenciar a disponibilidade de nutrientes, está relacionado a desequilíbrios fisiológicos da planta, enquanto alto teor de sais solúveis pode provocar a queima ou necrose das raízes, sendo resultante das condições inerentes do próprio substrato ou do excesso de adubação (BACKES et al., 1988; CARNEIRO, 1995).

Tabela 2-Condutividade elétrica (CE) e potencial hidrogeniônico $(\mathrm{pH})$ de Argissolo Vermelho-Amarelo distrófico (Arg), pó de coco (PC) e resíduo de sisal das combinações dos substratos para produção de mudas de sabiá. Recife,PE, 2004

Table 2 - Electrical Conductivity (EC) and Hydrogenionic Potential $(\mathrm{pH})$ of coir dust (CD) sisal residue $(S R)$ and distrofic red-yellow Argisoil (Arg) of substrate combinations for 'sabiá' seedling production. RecifePE, 2004

\begin{tabular}{lcc}
\hline Substratos & $\mathrm{CE}(\mathrm{dS} / \mathrm{m})$ & $\mathrm{pH}$ \\
\hline Arg* & $0,14 \mathrm{f}$ & $5,7 \mathrm{c}$ \\
PC* & $0,26 \mathrm{f}$ & $6,3 \mathrm{c}$ \\
RS $*$ & $2,91 \mathrm{a}$ & $9,3 \mathrm{a}$ \\
Arg+PC $(1: 1)$ & $0,15 \mathrm{f}$ & $6,5 \mathrm{c}$ \\
Arg+PC $(1: 2)$ & $0,11 \mathrm{f}$ & $6,6 \mathrm{c}$ \\
Arg+PC $(1: 4)$ & $0,17 \mathrm{f}$ & $6,7 \mathrm{c}$ \\
Arg+RS $(1: 1)$ & $1,16 \mathrm{e}$ & $8,1 \mathrm{~b}$ \\
Arg+RS $(1: 2)$ & $1,53 \mathrm{~d}$ & $9,1 \mathrm{ab}$ \\
Arg+RS $(1: 4)$ & $2,31 \mathrm{c}$ & $9,0 \mathrm{ab}$ \\
Arg+PC+RS $(1: 1: 1)$ & $1,65 \mathrm{~d}$ & $8,2 \mathrm{ab}$ \\
Arg+PC+RS $(1: 2: 2)$ & $2,18 \mathrm{c}$ & $8,6 \mathrm{ab}$ \\
Arg+PC+RS $(1: 2: 4)$ & $2,66 \mathrm{~b}$ & $8,7 \mathrm{ab}$ \\
Arg+PC+RS $(1: 4: 2)$ & $2,87 \mathrm{a}$ & $8,5 \mathrm{ab}$ \\
\hline CV $(\%)$ & 5,40 & 4,83 \\
\hline
\end{tabular}

Médias seguidas de mesmas letras na coluna não diferem estatisticamente a 5\% de probabilidade, pelo teste de Tukey. *Arg-solo, PC-pó de coco e RS-resíduo de sisal.
Com relação às variáveis de crescimento das mudas, como altura (h), diâmetro do coleto (D), número de folhas (NF), área foliar (AF) e matéria seca da parte aérea (MSPA) e os substratos RS, Arg+RS (1:1;1:4), Arg+PC+RS (1:2:4; e 1:4:2), não apresentaram número de repetições suficientes, pois as plântulas não alcançaram crescimento satisfatório, ocorrendo baixos porcentuais de sobrevivência, abaixo de $2 \%$, o que resultou na retirada desses quando se procederam às análises da variância para as variáveis morfofisiológicas estudadas (Tabela 3). A adição de resíduo de sisal às misturas, além de provocar baixo porcentual de sobrevivência das plântulas, afetou o crescimento das mudas de sabiá, sendo notório o efeito danoso desse resíduo. Observou-se, ainda, a rápida deterioração do resíduo de sisal logo que os recipientes foram preenchidos com as misturas de substratos.

Analisando a altura (h) das plântulas, verificouse que os substratos Arg+PC (1:1; 1:2 e 1:4) apresentaram médias superiores às dos demais, e o substrato Arg+RS (1:1) promoveu o menor crescimento das mudas em altura, ficando com $14,7 \mathrm{~cm}$ (Tabela 3). O mesmo foi verificado com relação aos substratos $\operatorname{Arg}+\mathrm{PC}+\mathrm{RS}(1: 1: 1$; e 1:2:2), com $45,0 \mathrm{~cm}$ e $36,3 \mathrm{~cm}$, respectivamente, isso devido, provavelmente, a Dp, CE e pH que apresentaram, conforme se pode observar nas Tabelas 1 e 2 .

Em termos de diâmetro do coleto (D), os substratos que se destacaram e obtiveram maiores médias foram Arg+PC (1:2), seguidos de PC, Arg+PC (1:1 e 1:4) e do substrato Arg, com um (D) de 5,4 mm. O menor valor observado foi o do substrato Arg+RS (1:1), de 2,0 mm, sendo significativamente diferente dos demais.

Tabela 3 - Altura das plantas (h), diâmetro do coleto (D), número de folhas (NF), área foliar (AF), matéria seca da parte aérea (MSPA) das mudas de sabiá cultivadas nos diferentes substratos. Recife-PE, 2004

Table 3 - Heigth plants (h), diameter (D), numbers of leaves $(N L)$, leaf area $(L A)$, dry matter of the arial part (MSPA) to sabiá seedlings produced in different substrates. Recife-PE, 2004

\begin{tabular}{|c|c|c|c|c|c|}
\hline Substratos & $\begin{array}{c}\mathrm{h} \\
\mathrm{cm}\end{array}$ & $\begin{array}{c}\mathrm{D} \\
\mathrm{mm}\end{array}$ & NF & $\begin{array}{l}\mathrm{AF} \\
\mathrm{cm}^{2}\end{array}$ & $\begin{array}{c}\text { MSPA } \\
\mathrm{g}\end{array}$ \\
\hline Arg* & $75,1 b$ & $5,4 b$ & $13,5 \mathrm{bcd}$ & $48,0 \mathrm{bc}$ & $11,7 \mathrm{cde}$ \\
\hline $\mathrm{PC}^{*}$ & $71,1 b$ & $5,9 \mathrm{ab}$ & $13,7 \mathrm{abcd}$ & $66,0 \mathrm{ab}$ & $13,1 \mathrm{bcd}$ \\
\hline Arg+PC $(1: 1)$ & $81,4 \mathrm{ab}$ & $5,7 \mathrm{ab}$ & $14,3 \mathrm{abc}$ & $62,3 \mathrm{ab}$ & $14,0 \mathrm{abc}$ \\
\hline Arg+PC $(1: 2)$ & $95,6 \mathrm{a}$ & $6,8 \mathrm{a}$ & $16,8 \mathrm{a}$ & $77,2 \mathrm{a}$ & $20,4 \mathrm{a}$ \\
\hline $\operatorname{Arg}+\mathrm{PC}(1: 4)$ & $80,1 \mathrm{ab}$ & $5,6 a b$ & $15,1 \mathrm{ab}$ & $69,5 \mathrm{a}$ & $19,2 \mathrm{ab}$ \\
\hline Arg+RS (1:1) & $14,7 \mathrm{~d}$ & $2,0 \mathrm{~d}$ & $10,4 \mathrm{e}$ & $9,1 \mathrm{~d}$ & $1,5 \mathrm{f}$ \\
\hline $\operatorname{Arg}+\mathrm{PC}+\mathrm{RS}(1: 1: 1)$ & $45,0 \mathrm{c}$ & $3,9 \mathrm{c}$ & $12,0 \mathrm{cde}$ & $41,8 \mathrm{c}$ & $7,1 \mathrm{def}$ \\
\hline Arg $+\mathrm{PC}+\mathrm{RS}(1: 2: 2)$ & $36,3 \mathrm{c}$ & $3,5 \mathrm{c}$ & $11,3 \mathrm{de}$ & $32,0 \mathrm{c}$ & $5,8 \mathrm{ef}$ \\
\hline $\mathrm{CV}(\%)$ & 10,15 & 9,34 & 6,0 & 13,97 & 20,05 \\
\hline
\end{tabular}

Médias seguidas de mesma letra na coluna não diferem estatisticamente entre si, pelo teste de Tukey a 5\% de probabilidade.*Arg-solo, PC-pó de coco e RS-resíduo de sisal.

R. Árvore, Viçosa-MG, v.30, n.2, p.163-170, 2006 
Analisando o número de folhas (NF) e a área foliar (AF), verificou-se que os substratos Arg+PC (1:2), Arg+PC $(1: 4 ; 1: 1)$ e PC obtiveram as maiores médias e não diferiram estatisticamente entre si, ficando a menor média por conta do substrato Arg+RS (1:1), diferindo, portanto, estatisticamente, de todos os demais, seguidos dos substratos Arg+PC+RS (1:2:2 e 1:1:1).

Com relação ao crescimento das mudas de sabiá, em termos de matéria seca da parte aérea, os substratos que obtiveram maiores médias foram $\operatorname{Arg}+\mathrm{PC}(1: 2 ; 1: 4$; e 1:1), os quais foram diferentes estatisticamente dos demais substratos testados, entretanto o substrato PC apresentou uma média próxima à desses tratamentos. A menor média foi obtida pelo tratamento Arg+RS (1:1), diferindo estatisticamente de todos os outros substratos avaliados, exceto os substratos Arg+PC+RS (1:2:2 e 1:2:14), conforme Tabela 3 .

Avaliando o crescimento de mudas de E. citriodora com diferentes combinações de substratos à base de pó de coco, composto orgânico, vermiculita e Plantmáx®, Pragana (1999) obteve valores médios maiores para número de folhas e valores médios menores com relação ao peso da matéria seca da parte aérea, em comparação com os desta pesquisa.

\section{CONCLUSÃO}

Nas condições deste experimento, os resultados permitiram a conclusão de que:

- Os substratos combinados contendo o pó de coco apresentaram melhores resultados com relação às características físicas e químicas, em comparação com os demais substratos, sendo recomendados para a produção de mudas de sabiá (Mimosa caesalpiniaefolia Benth).

- Recomenda-se a produção de mudas de sabiá (Mimosa caesalpiniaefolia Benth) com substratos à base de pó de coco ou quando este integrar mais de $50 \%$ das formulações em substratos contendo Argissolo Vermelho-Amarelo distrófico.

Não se recomenda a produção de mudas de sabiá (Mimosa caesalpiniaefolia Benth) contendo nas combinações dos substratos o resíduo de sisal.

\section{REFERÊNCIAS BIBLIOGRÁFICAS}

ANDRIOLO, J. L. et al. Caracterização e avaliação de substratos para o cultivo do tomateiro sem solo. Horticultura Brasileira, v. 17, n.3, p.215-219, 1999.
BACKES, M. A. et al. Substratos para a produção de plantas em viveiros. In: CONGRESSO FLORESTAL ESTADUAL, 1988, Nova Prata.

Anais...Nova Prata: Secretaria da Agricultura do Rio Grande do Sul, 1988. p. 665-675.

BENINCASA, M.M.P. Análise de crescimento de plantas: noções básicas. Jaboticabal: FUNEP, 1988. 42p.

BEZERRA, F. C. et al. Utilização de pó de coco como substrato de enraizamento para estacas de crisântemo. Revista Brasileira de Horticultura Ornamental, v. 7, n. 2, p.129-134, 2001.

BURGER, D. W.; HARTZ, T. K.; FORISTER, G. W. Composted green wast a container medium amendament for the production of ornamental plants. HortScience, v. 32, n. 1, p. 57-60, 1997.

CARneIRO, J.G.A Produção e controle de qualidade de mudas florestais. Curitiba: UFPR/FUPEP, 1995. 451p.

DE BOOTD, M.; VERDONCK, O. Physical properties of the substrates in horticulture. Acta Horticulture, v. 26, p. 37-44, 1972.

EMPRESA BRASILEIRA DE PESQUISA AGROPECUÁRIA - EMBRAPA. Manual de métodos de análise de solo. 2 ed. Rio de Janeiro: Ministério da Agricultura e do Abastecimento, 1997.212p.

FOnSECA, T. G. Produção de mudas de hortaliças em substratos de diferentes composições com adição de $\mathrm{CO}_{2}$ na água de irrigação. 2001. 72f. Dissertação (Mestrado em Agronomia) Escola Superior de Agricultura Luiz de Queiroz, Piracicaba, 2001.

FONTENO, W. C. Growing media types and physical/chemical proporties. In: REGD, D. W. (Ed). A growers guide to water, media and nutrition grenhouse crops. Batavia: Ball, 1996. p. 93-122.

GRÁS. R.; ÁGÜES, I. Quelques proprietés physiques dês substrats horticoles. Revue Horticole, n. 230, p. 789-791, 2000. Suplemento. 
GRAZIANO, T. T. et al. Interacão entre substratos e fetirrigacão na germinação e na produção de mudas Tagetes patula L. (compositae). Revista Brasileira de Horticultura Ornamental, v.1, n.2, p.78-85, 1995.

HANDRECH, K. A.; BLACK, N. Growing media for ornamental plants and flowers. Sydney: University of new South Wales Press, 1999. 448 p.

INGRAM, D. L.; HENLEY, R. W.; YEARGER, T. H. Diagnostic and monitoring produceres for nursey crops. Florida: University of Florida, 1990. 11p. (Bulletin, 556).

KAMPF, A. N. Produção comercial de plantas ornamentais. Guaíba: Agropecuária, 2000. 254p.

KÄMPF, A. N. Substratos para plantas: um desafio para a ciência do solo. Revista Opinião, v. 26, n.1, p. 5-16, 2001.

LORENZI, H. Árvores brasileiras: manual de identificação e cultivo de plantas arbóreas nativas do Brasil. Nova Odessa: Plantarum, 2002. 179p.

MINER, J. A. Substratos: propriedades y caracterizacion. Madri: Mundi Prensa, 1994. 172p.

MOREIRA, J. A. N. et al. Declínio do sisal e medidas para seu soerguimento no nordeste brasileiro. Campina Grande: EMBRAPA/CNPA, 1996. 19p. (Documentos, 45).

NEGREIROS, J. R. S. Diferentes substratos na formação de mudas de maracujazeiro-amarelo. Revista Ceres, v. 51, n. 294, p. 243-343, 2004.

NOGUERA, P. A. et al. Coconut coir waste, a new viable ecologilly - Friendly peat substitute. Acta Horticultural, v. 517, p, 279-286, 2000.

NORRIS, D. O.; DATE, R. A. Legume bacteriology. In: SHAW, N. H.; BRYAN, W. W. (Ed.) Tropical pastures research, principles and methods. Hurley: Commoweath Bureau of Pastures and Field Crops, 1976. p.134-174 (Bulletin, 51).

PAULILO, M. T. S.; FILIPPE, G. M. Crescimento de folhas de árvores de Qualeia grandifolia Mart. Revista Brasileira de Botânica, v.15, p. 85-93, 1992.

R. Árvore, Viçosa-MG, v.30, n.2, p.163-170, 2006
PRAGANA, R. B. Potencial de resíduo da extração da fibra de coco como substrato na produção agrícola. 1999. 86f. Dissertação (Mestrado em Ciência do Solo) - Universidade Federal Rural de Pernambuco, Recife, 1999.

PUCHALSHI, L. E. Sistema de produção de mudas em plug: propagação vegetativa de hibisco, Hibisco rosa-sinensis, L. 1999. $61 \mathrm{f}$. Dissertação (Mestrado em Agronomia) - Universidade Federal do Rio Grande do Sul, Porto Alegre, 1999.

RODRIGUES. J. J. L. et al. Características físicas de substrato horticola. In: CONGRESSO BRASILEIRO DE CIÊNCIA DO SOLO, 25. 1995, Viçosa, MG. Resumos...Viçosa, MG: Sociedade Brasileira de Ciência do Solo, 1995. v.1, p.189.

SANTOS, K.S.R. Atuação do enxofre com Thiobacillus na solubilização do fosfato natural e materiais orgânicos em solo de tabuleiro cultivado com sabiá (Mimosa caesalpiniaefolia Benth) 2002. 63f. Dissertação (Mestrado em Ciência do Solo). Universidade Federal Rural de Pernambuco, Recife, 2002.

SCHIE, W. van. Standardization of substrates. Acta Horticulturae, v.1, n. 481, p. 71-77, 1999.

SILVEIRA, R.L.V. et al. Adubação e nutrição de espécies nativas: viveiro e campo. São Paulo: Universidade São Paulo, 2002. 22p.

SOUZA, M. M. et al. Avaliação de substratos para o cultivo de crisântemo (Chrysanthemum morifolium Ramat., compositae) 'white polaris' em vasos.

Revista Brasileira de Horticultura e Ornamental, v. 1, n.2, p. 71-77, 1995.

TEO, C.K.H; TAN, E.H. Tomato production in cocopeat. Planter, v.69 p.239-242, 1993.

TILLMANN, M.A.A. et al. Comparação entre diversos substratos no enraizamento de estacas de cróton (Codiaceum variegatum L.) Scientia Agrícola, v.51, n.1, p.17-20, 1994.

VERDONCK, O. Reviewing and evaluation of new materiales used as susbstrates. Acta Horticulturae, v. 150, p. 155-160, 1984.

WILSON, C. G. S. Tomato production in bark substrates. Acta Horticulturae, v. 150, p.271-276, 1983. 